\title{
Rehabilitation of injured U.S. servicemember with traumatic brain injury, stroke, spinal cord injury, and bilateral amputations: A case report
}

\author{
Ajit B. Pai, MD; ${ }^{1-2 *}$ Nicholas R. Jasper, MD; ${ }^{3}$ David X. Cifu, MD $^{2,4}$ \\ ${ }^{1}$ Physical Medicine and Rehabilitation Service, Hunter Holmes McGuire Department of Veterans Affairs Medical Cen- \\ ter, Richmond, VA; ${ }^{2}$ Department of Physical Medicine and Rehabilitation, Virginia Commonwealth University, Rich- \\ mond, VA; ${ }^{3}$ Orthopaedic \& Spine Specialists-Physical Medicine and Rehabilitation, York, PA; ${ }^{4}$ Physical Medicine and \\ Rehabilitation Services, Veterans Health Administration, Richmond, VA
}

\begin{abstract}
The complexity of injuries that can arise from combat situations, specifically as a result of the Operation Iraqi Freedom/Operation Enduring Freedom conflicts, is challenging. As injured troops leave the war zone early for medical care, they are treated by an interwoven system of care that includes both the Department of Defense and Veterans Health Administration. Physicians across these settings are increasingly faced with patients who have multiple rehabilitation diagnoses as a result of the severity of blast injuries; therefore, the importance of active communication across the system must be highlighted. This case describes the seamless transition of an injured soldier across the continuum of care.
\end{abstract}

Key words: Active Duty, amputation, Department of Defense, Department of Veterans Affairs, polytrauma, rehabilitation, spinal cord injury, stroke, traumatic brain injury, veteran.

\section{INTRODUCTION}

On October 7, 2001, the United States began combat operations in Afghanistan, referred to as Operation Enduring Freedom (OEF), in response to the September 11 terrorist attacks. On March 2003, the United States became engaged in military operations in Iraq, referred to as Operation Iraqi Freedom (OIF), as part of the Global War on Terror. OIF was completed as of August 31, 2010, and transitioned to Operation New Dawn (OND) as of September 1, 2010. The OEF/OIF/OND conflicts have been the longest, continuous military campaign in the history of the United States, with more than 2.2 million

\begin{abstract}
Abbreviations: ACA $=$ anterior cerebral artery, AIS $=$ American Spinal Injury Association Impairment Scale, AKA = above-knee amputation, ASIA = American Spinal Injury Association, $\mathrm{BKA}=$ below-knee amputation, $\mathrm{C}$-collar = cervical collar, $\mathrm{CT}=$ computed tomography, DOD $=$ Department of Defense, FIM = Functional Independence Measure, GCS = Glasgow Coma Scale, HHMVAMC = Hunter Holmes McGuire Department of Veterans Affairs Medical Center, IED = improvised explosive device, JTTS $=$ Joint Theater Trauma System, L = lumbar, LRMC = Landstuhl Regional Medical Center, $\mathrm{MCA}=$ middle cerebral artery, $\mathrm{NNMC}=$ National Naval Medical Center-Bethesda, OEF $=$ Operation Enduring Freedom, OIF $=$ Operation Iraqi Freedom, OND $=$ Operation New Dawn, PRC = Polytrauma Rehabilitation Center, PSC = Polytrauma System of Care, RLAS = Rancho Los Amigos Scale, $\mathrm{SCI}=$ spinal cord injury, $\mathrm{T}=$ thoracic, $\mathrm{TBI}=$ traumatic brain injury, VA = Department of Veterans Affairs, VHA = Veterans Health Administration, WRAMC = Walter Reed Army Medical Center, WRNMMC = Walter Reed National Military Medical Center.

*Address all correspondence to Ajit B. Pai, MD; Physical Medicine and Rehabilitation Service, Hunter Holmes McGuire VA Medical Center, 1201 Broad Rock Blvd, PM\&RS (652/117), Richmond, VA 23249; 804-675-5000, ext 3232; fax: 804-675-6989. Email: ajit.pai@va.gov
\end{abstract}

http://dx.doi.org/10.1682/JRRD.2011.11.0224 
troops deployed as of December 2010. ${ }^{*}$ These conflicts have spawned more powerful explosive devices that use creative detonation methods and carry devastating blast components, leading to multiple organ system injuries, known as the polytrauma condition. Blast injury from explosive forces has proven to be the most devastating injury seen in OIF/OEF. The pervasiveness of blast exposures, especially in the form of improvised explosive devices (IEDs), and their impact on the brain have resulted in traumatic brain injury (TBI) being referred to as the "signature wound" of this war [1]. Fortunately, despite these tactics, the ability of the U.S. military's trauma teams to rapidly treat, stabilize, and aeromedically evacuate servicemembers with severe injuries has resulted in an increased survivability compared with the Vietnam war [2].

This case presentation describes a combat-injured servicemember with highly complex rehabilitation needs who was successfully returned to the community. To date, no other cases with such severe injuries in one individual have been described in the literature (search parameters included amputation, stroke, spinal cord injury [SCI], and TBI). This case demonstrates the level of coordination required to seamlessly navigate the combined Department of Defense (DOD) and Department of Veterans Affairs (VA) system and the vital role that rehabilitation services may play in optimal recovery after blast injury.

\section{CASE REPORT}

E.M. is a 32-year old, right-hand dominant, previously healthy medic in the U.S. Army who sustained an IED blast on July 2, 2010, while stationed in Afghanistan as part of OEF. As a result of the blast, he experienced the following injuries:

1. Severe TBI with multiple facial fractures, including the right pterygoid process lateral plate, the right parasymphyseal mandible, the left maxillary sinus medial wall, and the nasal septum.

\footnotetext{
*Deployment File Baseline Report Contingency Tracking System; Defense Manpower Data Center. Provided to Employee Education System by the Armed Force Health Surveillance Center; February 16, 2011.
}

2. Right middle cerebral artery (MCA) distribution infarction.

3. Bilateral anterior cerebral artery (ACA) watershed infarctions.

4. Traumatic, bilateral below-knee amputations (BKAs).

5. Lumbar (L) 1 American Spinal Injury Association (ASIA) Impairment Scale (AIS)-B SCI from an unstable, comminuted burst fracture of L4 with retropulsed fracture, resulting in 30 percent effacement of the central canal [3].

6. L1-L2 superior endplate compression fracture.

7. Left transverse process fractures of L1, L2, L3, and L4, with associated retroperitoneal, paravertebral hematomas.

8. Coccyx fracture.

9. Right lung middle and posterior segment lobe contusions with hematoma.

He had an initial loss of consciousness in the field following the blast, became alert and agitated while being aeromedically evacuated to the Bagram Airfield military hospital (Bagram, Afghanistan), and was noted to be comatose with a Glasgow Coma Scale (GCS) of 6 on arrival at Bagram [4]. He was intubated, sedated, and placed in a cervical collar (C-collar). Initial head computed tomography (CT) scan was normal. A diagnostic peritoneal lavage was negative. There was no detectable blood flow to his lower limbs, and therefore, he underwent surgical revision of the bilateral BKAs. A postoperative head CT was significant for a right MCA infarction. On July 4, he was aeromedically evacuated to Landstuhl Regional Medical Center (LRMC) (Landstuhl, Germany). Before transfer, the patient was discussed during the Joint Theater Trauma System (JTTS) weekly call and his physician documented pertinent information in the Joint Patient Tracking Application. The JTTS weekly call is conferenced to military treatment facilities across the transfer route, including LRMC; Walter Reed National Military Medical Center (WRNMMC), which at the time was split into Walter Reed Army Medical Center (WRAMC) and National Naval Medical CenterBethesda (NNMC); and VA Polytrauma Rehabilitation Centers (PRCs). In addition, within hours of the initial injury, the patient's family was notified of his injury. In this patient's case, his wife, an Army medic herself, was at his bedside within 4 hours.

Due to concern over a right internal carotid artery dissection, he was started on anticoagulation. A follow-up CT scan demonstrated no evidence of bleeding, edema, or midline shift, but did reveal evolution of the right 
MCA infarction, plus a new right MCA-ACA watershed and left posterior frontoparietal infarction. A Codman Bolt (DePuy Orthopaedics, Inc; Warsaw, Indiana) was placed for intracranial pressure monitoring on July 5.

He was then aeromedically evacuated to WRAMC on July 6 while still intubated and sedated. Again, the patient was discussed on the JTTS weekly conference call, and the primary surgical team spoke to the family to complete the loop of communication. From a medical standpoint, anticoagulation was continued for presumed carotid dissection. Keppra was begun for seizure prophylaxis. On the morning of July 7, he underwent washouts of his bilateral BKAs. He was found to have gram-negative rods and cocci on admission peripheral blood cultures, and therefore, he was started on intravenous antibiotics. Neurologically, he did not readily awaken and improve with the weaning off of sedation. An angiogram showed cerebral vasospasm, with decreased flow in the distributions noted.

He was transferred to NNMC on July 8 for management of his neurovascular issues. Before this transfer, the surgeons at WRAMC spoke via telephone to the surgeons at NNMC to discuss medical concerns. In turn, they spoke with the appropriate case managers at their respective facilities to coordinate the transfer. These decisions were discussed with the patient's family before transfer. Upon arrival to NNMC, the patient had a GCS of 6, was on a ventilator, and had a C-collar and bilateral lowerlimb wound drains in place. He had spontaneous nonpurposeful movement in the right upper limb and withdrew to painful stimuli, but had no eye opening. He underwent revisions of his bilateral BKAs to definitive above-knee amputations (AKAs) on July 16 as a result of poor circulation, persistent infection, and nonhealing wounds.

The patient was noted to begin making more purposeful movements by July 24 and was also able to be extubated; he was noted to be out of coma because he was consistently following commands [5]. By this time, he was noted to have a persistent moderate left hemiplegia (poor strength proximally and trace strength distally of upper limb), difficulty with visual tracking, bowel and bladder incontinence, and functional dependence. He was initiated in active rehabilitation therapies, and plans for transition to the VA Polytrauma System of Care (PSC) were made. These plans included (1) video teleconferences between the managing team at NNMC and the rehabilitation team at the Hunter Holmes McGuire VA Medical Center (HHMVAMC) in Richmond, Virginia;
(2) video teleconferences between the patient's wife and the rehabilitation team at HHMVAMC; and (3) a visit to HHMVAMC by the patient's wife. Over the next several weeks, the patient began to demonstrate increasing cognitive skills and engagement with the treating team. He reported left elbow pain but had normal bone imaging, and the pain was managed with heat, ice, and therapy care. He demonstrated the ability to safely swallow, but he was unable to meet his needs orally; therefore, gastrostomy tube feedings were continued. With his increasing sitting time in bed and need to spend time out of bed, neurosurgery reassessed his L4 burst fracture. It was initially managed with logrolling in bed, a thoracolumbosacral orthosis restriction for out of bed, a bone stimulator, and a progressive sitting program. The HHMVAMC polytrauma team recommended a more in-depth assessment of the integrity of his spinal cord, given his increased awareness and mobilization. While assessment was limited by his bilateral AKAs, he was found on initial examination to have L1 AIS B paraplegia. This was diagnosed by sensory examination with light touch (cotton wisp) and pinprick (safety pin). In addition, the motor examination focused on the right side where the patient still had 3/5 (by manual motor testing) motor strength in the hip flexors. Although this examination was difficult because of hemiparesis from his cerebral infarct, sensory examination showed normal sensation bilaterally in both light touch and pinprick down to L1 with intact sacral sensation, but no voluntary anal sphincter contraction. In light of this and because of worsening L4 protrusion on imaging, neurosurgery performed an L3-L5 laminectomy, duraplasty, and L2-sacral 1 fusion on September 18.

The patient was transferred to the HHMVAMC PRC in Richmond, Virginia, for comprehensive inpatient brain injury rehabilitation on September 30. Upon admission, he met his treatment team, which consisted of the physiatrist, polytrauma fellow, physiatry resident, nursing staff, physical therapist, occupational therapist, speechlanguage pathologist, kinesiotherapist, recreational therapist, blind rehabilitation specialist, counseling psychologist, neuropsychologist, dietician, social work case manager, Army liaison, and family/patient educator. The SCI and Amputation teams of the VA PSC were involved in the rehabilitation process. His admission evaluation was significant for a benign general medical survey, with the following focal issues: mild confusion, but appropriate behavior (Rancho Los Amigos Scale [RLAS] level VI) and memory deficits; a more thorough ASIA evaluation 
demonstrating T12 AIS B paraplegia (original AIS examination was completed by a non-SCI board-certified physiatrist; upon admission to the PRC, an AIS was completed by an SCI board-certified physiatrist) as patient had reduced pinprick sensation in the L1 dermatome; left hemiplegia (moderate weakness of proximal left arm and leg, severe weakness of distal left arm); hoarseness; healed coccygeal skin ulcer; small, clean right distal AKA wound; persistent bilateral AKA pain (managed with scheduled oral morphine, memantine, and pregabalin); indwelling Foley catheter; and functional dependency (Functional Independence Measure [FIM] of 40) [6-7]. He was initiated in a full program and slowly progressed in all areas of disability and function. He remained in the program for 3.5 mo. In addition to traditional interdisciplinary TBI rehabilitation programming, elements of SCI medicine (bowel and bladder programs, skin checks and care, weekly education) and amputation care (preprosthetic training, residual-limb preparation) were used. Before discharge, the patient and his spouse successfully participated in multiple therapeutic outings, which enabled them to complete an extended therapeutic pass in New England to witness the return of his unit.

At the time of discharge, the patient was at an RLAS level of VIII (comprehensive neuropsychological assessment revealed mild persistent deficits in executive functioning); had a FIM score of 61; had L1 AIS B paraplegia (intact light touch and pinprick in the L1 dermatome, but reduced below the level of injury) with lower motor neuron areflexic bladder and bowel function; demonstrated good strength ( $3 / 5$ by manual muscle testing) across the left shoulder, elbow, and wrist with trace strength of the hand; and had an 8-10 h out-of-bed and sitting-time tolerance. He was transitioned to the Boston VA Medical Center for a short medical stay before living in an offcampus apartment there to allow for daily outpatient SCI rehabilitation services and to be closer to home. Before transfer, a warm handoff via video teleconference was initiated with the accepting team. His discharge equipment included residual-limb shrinkers, two triangular bed wedges, a left wrist and finger dorsal resting hand splint, a hemisling, a power tilt/recline/elevate wheelchair, a skin inspection mirror, and a Bioness (Valencia, California) hand functional electrical stimulation unit. His medications were significant for scheduled pain medications (amitriptyline, pregabalin); as needed pain medication for use with therapy (oxycodone); a scheduled sleep medication (quetiapine) originally prescribed for agitation reduction overnight, but continued as a sleep aid since he did not experience any adverse effects and requested the continued use; and bowel program agents.

Four months after his transfer to the Boston VA Medical Center, he transitioned back to the DOD at the Center for the Intrepid, San Antonio Military Medical Center, in San Antonio, Texas. Before transfer, the Boston VA case manager and military case manager established ongoing communication. This allowed for teams at both facilities to coordinate treatment plans. These interactions culminated in a teleconference between the two teams before the patient's transfer, which allowed for answering of questions and attenuation of patient and family anxiety surrounding the transition. He is currently enrolled in a state-of-the-art amputee rehabilitation program with the goal of ambulating independently on prosthetics.

\section{DISCUSSION}

This case illustrates the complexity of injuries that can arise from combat situations, specifically as a result of the OIF/OEF conflicts. As injured troops leave the forward theater early for stabilization, they are treated by an interwoven system of care that includes both the DOD and the Veterans Health Administration (VHA). As physicians across these settings are increasingly faced with patients who have multiple rehabilitation diagnoses as a result of the severity of blast injuries, the importance of active communication across the system must be highlighted. Video teleconferences, shared radiologic imaging databases, and scheduled telephone conversations allow for increasingly seamless transition between the various care sites. These tools also allow for timely consultation between clinicians across sites, which reduces delays in treatment and the need for multiple transfers.

In 2004, Congress passed two laws that required the development of a specialized system of care to meet the complex rehabilitation needs of servicemembers and veterans injured in combat operations. As noted, while TBI remains the "signature injury" in polytrauma, combat injuries also include SCI, amputation, complex fracture and other musculoskeletal trauma, burns, and psychological difficulties. The VHA implemented the requirements of public laws passed in 2004 (Section 302 of Public Law 108-422 and Public Law 108-447) by developing the PSC, which integrates specialized rehabilitation services available at regional centers, network sites, and local VA 
medical centers [8-9]. The VA's nationwide SCI Centers, the Amputation System of Care, the Assistive Technology Centers of Excellence, and a virtual Polytrauma Telehealth Network supplement the core PSC network. Additionally, an integrated system of nationwide VADOD collaborative case management was established; VA rehabilitation nurse liaisons are embedded in the DOD's WRNMMC and San Antonio Military Medical Center, and military liaisons are present in many of the VA polytrauma sites. The VA Polytrauma and TBI rehabilitation care mission is to provide state-of-the-art care to manage the patient's rehabilitation, physical, and mental health needs while being as close as possible to the patient's home or family support. This PSC matrix is dedicated to providing rehabilitation services that restore physical, intellectual, communicative, psychosocial, and vocational skills and to facilitating the transfer of those skills from the hospital setting to daily life in the home community. These services include, but are not limited to, inpatient rehabilitation, outpatient rehabilitation, emerging consciousness programs, transitional or residential rehabilitation, day programs, vocational rehabilitation, driver's training, and community reentry programs. The PSC also manages the ongoing and emerging rehabilitation and psychosocial needs of veterans with polytrauma and TBI. This includes on-going follow-up and treatment; life-long medical care; case management with monitoring and implementation of the treatment plan; coordination of services between the VA, $\mathrm{DOD}$, and private sector; oversight of the quality and intensity of VA and non-VA services; and provision of education and support for patients and caregivers. Research, education, and training of the next generation of clinicians are also key elements of the overall PSC.

In addition to the comprehensive, interdisciplinary services inherent in Commission on the Accreditation of Rehabilitation Facilities-accredited inpatient TBI rehabilitation programs, the VA PSC programs have additional resources in place during both the inpatient and residential phases of care focused on community reintegration and vocational rehabilitation. These services, along with the availability of specialized benefits to finance advanced education (i.e., college courses), are additional resources in place for Active Duty military and serviceconnected veterans from OEF/OIF/OND that enhance the rehabilitation experience and facilitate a smoother transition back to productivity, including continued military service. Although this patient did not feel ready to partici- pate in a college course or vocational program during his acute inpatient rehabilitation stay, he was excited at the prospect of having this available to him in the future. Among the PSC rehabilitation program adjustments available to these individuals are liberal policies on therapeutic leaves, which are used to assist patients and families who may have homes on or near military bases that may be hundreds or even thousands of miles away from the VA PRC site. To facilitate travel to these often distant home sites, therapeutic passes may be extended for several nights in a row and/or several weekends in a row, as needed. During these passes, a patient and his or her family become more aware of both the patient's abilities and the challenges that they will face once they leave the inpatient rehabilitation setting. In this particular case, this patient's extended pass in New England allowed him and his spouse to understand the barriers to returning home. Awareness of these challenges allows a patient and the involved family to help further direct their rehabilitative needs-whether it is trouble with car or bed transfers, difficulty performing intermittent catheterizations, or bowel program confusion. Experiences on passes may also help determine what types of equipment a patient may need upon final discharge from the inpatient rehabilitation setting-ranging from simple tub benches, to functional orthoses for eating or catheterizations, to hospital beds, or even to home modifications. Vocational rehabilitation, educational programs, therapeutic leaves, and transitional living can help build a patient's selfesteem and confidence and, ultimately, foster a return to independence. In the case of this patient, the outcome was on par with his expectations. Initially, his goal was to regain the ability to walk with prostheses, but this goal was tempered throughout his rehabilitation course and supplanted with other more attainable goals for the purpose of acute rehabilitation. Those goals focused on improved cognitive performance, power wheelchair mobility, pressure reliefs, bowel and bladder management, and increased independence with basic self-care.

Within each PSC site, the integrated system of care includes TBI, SCI, amputation, pain, and assistive technology specialties under the supervision of the physical medicine and rehabilitation physician. Without the collaboration of these rehabilitative specialties, much of the complex medical and rehabilitation care could not be managed. As the system has integrated each of these specialty areas and personnel at the sites, the goals of successfully caring for complex medical-surgical conditions and obtaining functional independence can be 
achieved more effectively. In the case of this soldier, despite his multiple impairments and medical diagnoses, he was able to achieve greater functional independence with the assistance of family supports and continue therapy in an outpatient setting. For many of our troops, functional independence can mean returning to duty or to their families. The interwoven VA PSC and the DOD care network represents an example of the successful application of interdisciplinary care across multiple medical sites to rehabilitate America's wounded warriors.

\section{CONCLUSIONS}

Servicemembers injured during the OEF/OIF/OND conflicts often times require intensive care through the spectrum of DOD and VA services. Injury patterns seen include TBI, amputations, SCI, stroke, and complex orthopedic and soft tissue injuries. In order to treat these patients, it is imperative that physicians across institutions understand the medical care undertaken at each institution along the continuum. Thus, the importance of communication among providers cannot be overstated.

\section{ACKNOWLEDGMENTS}

\section{Author Contributions: \\ Drafting of manuscript: A. B. Pai, N. R. Jasper, D. X. Cifu. Critical revision of manuscript: A. B. Pai. \\ Administrative, technical, or material support: A. B. Pai.}

Financial Disclosures: The authors have declared that no competing interests exist.

Funding/Support: This material was unfunded at the time of manuscript preparation.

Additional Contributions: Dr. Jasper is now affiliated with the Department of Physical Medicine and Rehabilitation, Indiana University, Indianapolis, Indiana.

Participant Follow-Up: The authors have informed the participant of the future publication of this study.

\section{REFERENCES}

1. Martin EM, Lu WC, Helmick K, French L, Warden DL. Traumatic brain injuries sustained in the Afghanistan and Iraq wars. J Trauma Nurs. 2008;15(3):94-99, quiz 100-101. [PMID:18820555]

2. Meyer KS, Marion DW, Coronel H, Jaffee MS. Combatrelated traumatic brain injury and its implications to military healthcare. Psychiatr Clin North Am. 2010;33(4):783-

\section{6. [PMID:21093678]}

http://dx.doi.org/10.1016/j.psc.2010.08.007

3. Ditunno JF Jr, Young W, Donovan WH, Creasey G. American Spinal Injury Association. The international standards booklet for neurological and functional classification of spinal cord injury. Paraplegia. 1994;32(2):70-80.

[PMID:8015848]

http://dx.doi.org/10.1038/sc.1994.13

4. Teasdale G, Jennett B. Assessment of coma and impaired consciousness. A practical scale. Lancet. 1974;2(7872):8184. [PMID:4136544] http://dx.doi.org/10.1016/S0140-6736(74)91639-0

5. Whyte J, Cifu D, Dikmen S, Temkin N. Prediction of functional outcomes after traumatic brain injury: a comparison of 2 measures of duration of unconsciousness. Arch Phys Med Rehabil. 2001;82(10):1355-59. [PMID:11588737] http://dx.doi.org/10.1053/apmr.2001.26091

6. Hagen C, Malkmus D, Durham P, Bowman K. Levels of cognitive functioning. In: Rehabilitation of the head injured adult: comprehensive physical management. Downey (CA): Professional Staff Association of Rancho Los Amigos Hospital; 1979.

7. Granger CV, Hamilton BB, Sherwin FS. Guide for use of the Uniform Data Set for medical rehabilitation. Buffalo (NY): State University of New York; 1986.

8. Department of Veterans Affairs. Polytrauma/TBI System of Care [Internet]. Washington (DC): Department of Veterans Affairs. Available from: http://www.polytrauma.va.gov/

9. Cifu DX, McNamee S, Gater D, Walker WC, Ericksen J, Murphy D, Oliver M. The Polytrauma Rehabilitation System of Care programs at the Richmond Veterans Administration Medical Center. Crit Rev Phys Rehabil Med. 2009; 21(3-4):197-213.

http://dx.doi.org/10.1615/CritRevPhysRehabilMed.v21.i3-4.10

Submitted for publication November 23, 2011. Accepted in revised form January 30, 2012.

This article and any supplementary material should be cited as follows:

Pai AB, Jasper NR, Cifu DX. Rehabilitation of injured U.S. servicemember with traumatic brain injury, stroke, spinal cord injury, and bilateral amputations: A case report. J Rehabil Res Dev. 2012;49(8):1191-96. http://dx.doi.org/10.1682/JRRD.2011.11.0224

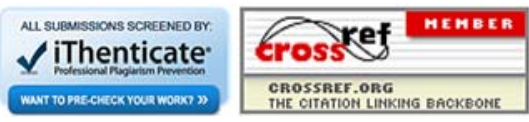

